\title{
Edge and Contrast Enhancement Using Spatially Incoherent Correlation Holography Techniques
}

\author{
Vijayakumar Anand ${ }^{1, *(\mathbb{D}}$, Joseph Rosen ${ }^{2}{ }^{(}$, Soon Hock $\mathrm{Ng}^{1}{ }^{1}$, Tomas Katkus ${ }^{1}$, Denver P Linklater ${ }^{3}{ }^{(}$, \\ Elena P Ivanova ${ }^{3}$ and Saulius Juodkazis ${ }^{1,4}$ (D) \\ 1 Optical Sciences Centre and ARC Training Centre in Surface Engineering for Advanced Materials (SEAM), \\ School of Science, Swinburne University of Technology, Hawthorn, VIC 3122, Australia; \\ soonhockng@swin.edu.au (S.H.N.); tkatkus@swin.edu.au (T.K.); sjuodkazis@swin.edu.au (S.J.) \\ 2 School of Electrical and Computer Engineering, Ben-Gurion University of the Negev, P.O. Box 653, \\ Beer-Sheva 8410501, Israel; rosenj@bgu.ac.il \\ 3 School of Science, RMIT University, Melbourne, VIC 3000, Australia; denver.linklater@rmit.edu.au (D.P.L.); \\ elena.ivanova@rmit.edu.au (E.P.I.) \\ 4 Tokyo Tech World Research Hub Initiative (WRHI), School of Materials and Chemical Technology, \\ Tokyo Institute of Technology, 2-12-1, Ookayama, Meguro-ku, Tokyo 152-8550, Japan \\ * Correspondence: vanand@swin.edu.au
}

Citation: Anand, V.; Rosen, J.; Ng, S.H.; Katkus, T.; Linklater, D.P.; Ivanova, E.P.; Juodkazis, S. Edge and Contrast Enhancement Using Spatially Incoherent Correlation Holography Techniques. Photonics 2021, 8, 224. https://doi.org/ $10.3390 /$ photonics 8060224

Received: 19 May 2021

Accepted: 15 June 2021

Published: 16 June 2021

Publisher's Note: MDPI stays neutral with regard to jurisdictional claims in published maps and institutional affiliations.

Copyright: (c) 2021 by the authors. Licensee MDPI, Basel, Switzerland. This article is an open access article distributed under the terms and conditions of the Creative Commons Attribution (CC BY) license (https:// creativecommons.org/licenses/by/ $4.0 /)$.

\begin{abstract}
Image enhancement techniques (such as edge and contrast enhancement) are essential for many imaging applications. In incoherent holography techniques such as Fresnel incoherent correlation holography (FINCH), the light from an object is split into two, each of which is modulated differently from one another by two different quadratic phase functions and coherently interfered to generate the hologram. The hologram can be reconstructed via a numerical backpropagation. The edge enhancement procedure in FINCH requires the modulation of one of the beams by a spiral phase element and, upon reconstruction, edge-enhanced images are obtained. An optical technique for edge enhancement in coded aperture imaging (CAI) techniques that does not involve two-beam interference has not been established yet. In this study, we propose and demonstrate an iterative algorithm that can yield from the experimentally recorded point spread function (PSF), a synthetic PSF that can generate edge-enhanced reconstructions when processed with the object hologram. The edge-enhanced reconstructions are subtracted from the original reconstructions to obtain contrast enhancement. The technique has been demonstrated on FINCH and CAI methods with different spectral conditions.
\end{abstract}

Keywords: edge enhancement; contrast enhancement; vortex filter; Fresnel incoherent correlation holography; coded aperture imaging

\section{Introduction}

Image enhancement methods have played a crucial role in identifying the key features of an image and for increasing the contrast in X-ray imaging [1], ultrasound imaging [2], synthetic aperture radar (SAR) imaging [3], and many other imaging applications. While edge enhancement is preferred in many applications, in some cases (such as SAR), edge enhancement is critically essential, as SAR data are highly heterogenous, unlike regular image data, and without edge enhancement, the detection rates cannot reach acceptable values [3]. There are different techniques to perform edge enhancement. One well-established method is using Fourier optics, where only the higher spatial frequencies responsible for the edge information are allowed and the lower spatial frequencies are blocked [4]. Another widely used method is the use of vortex filters to enhance the edge information [5-8]. The above approach has also been successfully implemented in incoherent three-dimensional (3D) imagers.

Fresnel incoherent correlation holography $(\mathrm{FINCH})$ is a super-resolution imaging method used in many applications [9-12]. In FINCH, the light from any object point is 
split into two and modulated differently by two quadratic phase masks (QPMs) with different focal distances while both beams are interfered to create the incoherent hologram. FINCH, in the in-line configuration, requires at least three camera shots with relative phase shifts to function, and a complex-valued hologram is generated by projecting the recorded holograms into the complex space. The 3D image of the object is reconstructed by numerically propagating the complex hologram to the image plane. The edge enhancement study in FINCH was first demonstrated by Bouchal and Bouchal using the vortex filter [13], and the system has been called spiral-FINCH (S-FINCH). In S-FINCH, one of the object waves was modulated by a spiral phase mask (SPM) while the other was modulated by a QPM, and by numerical backpropagation, the reconstructed holograms generated edge-enhanced images. The principle of operation may be understood as follows; In SFINCH, every object point undergoes a spiral phase modulation, and it interferes with the same point after quadratic phase modulation. The resulting hologram, when numerically backpropagated to the focal distance of the QPM, generates a donut-shaped spot [14]. Therefore, for a complicated object, edge enhancement is obtained during reconstruction.

Based on the above studies, edge enhancement was implemented for FINCH microscopy with fundamental order [15] and higher-order vortex filters [16]. In all of the above studies $[13,15,16]$, a two-beam interference was needed. Hence, the edge enhancement procedure was direct where the vortex filter modulation was introduced to one of the interfering beams, and the numerical reconstruction was carried out by a Fresnel backpropagation. Recently, there was a rapid advancement in incoherent holography techniques without two-beam interference. Instead of modulating two beams, only a single beam is scattered via a chaotic phase mask [17-22]. In the above interferenceless approaches, it is necessary to record the point spread function (PSF) of the system and to reconstruct the 3D image by various reconstruction methods (such as phase-only matched filter [17], Weiner filter [23], and Lucy-Richardson algorithm [24]). In the above cases [17-22], the technique proposed in [13] cannot be applied because they lack two-beam interference. Another aspect of the above vortex filtering approach is that the method also demands resources such as a spiral phase plate that is either manufactured or displayed on an active device (such as a spatial light modulator (SLM)). When computational optical techniques are able to efficiently simplify the optical experiments with lesser optical elements and fewer camera shots, the use of SLM or fabrication of micro-optical elements makes the system complicated.

Numerical and experimental studies have been carried out in FINCH [25-27] with a modified reconstruction method that does not involve a numerical backpropagation, but rather a cross-correlation with the recorded PSF, similar to [17-19]. Unlike the previous studies such as S-FINCH, where the reconstructing process is independent of the modulation function, the cross-correlation-based approach uses a reconstructing function that is dependent upon the modulating functions. Consequently, the modulation function is not expressed during the reconstruction [27]. In $\mathrm{FINCH}$, the reconstruction function is the same irrespective of the type of modulation used: whether it is a quadratic phase or a spiral phase modulation, it is always numerical backpropagation. Therefore, relative variations (such as edge enhancement) can be easily obtained. In coded aperture imaging (CAI) methods, the reconstructing function (PSF) is dependent upon the modulation function, and so during reconstruction by cross-correlation, the characteristics of the modulations are suppressed [27].

In this study, we propose a computational optical technique for engineering the PSF for edge enhancement in interferenceless imaging techniques and in FINCH with reconstruction by cross-correlation. In general, in linear optical systems, the recorded PSF of the system is sufficient to fully understand its imaging characteristics. In this study, a synthetic PSF is generated from the experimentally recorded PSF using an iterative algorithm such that the reconstructed image of the object is edge-enhanced. The manuscript consists of four sections. The methodology and the iterative algorithm are discussed in the next section. The experimental results are presented in the third section. The results are 
discussed in the fourth section, and the method and its future perspectives are summarized in the final section.

\section{Methods}

A generalized optical configuration is considered, which includes many possibilities: light emitted from every point of an object is modulated by a mask, and its intensity is recorded [17-22]. Alternatively, light is split into two beams [9-12,28] and coherently interfered, as shown in Figure 1. The optical imaging system is linear but optionally includes refractive lenses, diffractive optical elements, diffractive lenses, and other optical components. The proposed method is suitable for all incoherent linear shift-invariant imaging systems that use an indirect imaging approach using cross-correlation with the PSF. For a point object at the object plane $(x, y)$, the intensity of the point spread hologram (PSH) $I_{\mathrm{PSH}}(u, v)$ is the intensity impulse response of the system. For a multi-point $2 \mathrm{D}$ object with an intensity distribution $t(x, y)$, the system intensity response is $I_{t}(u, v)=I_{\mathrm{PSH}}(u, v) \oplus t(x, y)$, where ' $\oplus$ ' is a $2 \mathrm{D}$ convolution operator. The image of the object can be reconstructed as $I_{R}\left(x^{\prime}, y^{\prime}\right)=I_{t}(u, v) * I_{\mathrm{PSH}}(u, v)$, where "*" is a $2 \mathrm{D}$ correlation operator. Substituting the expression of $I_{t}$ in $I_{R}, I_{R}\left(x^{\prime}, y^{\prime}\right)=I_{\mathrm{PSH}}(u, v) \oplus t(x, y) * I_{\mathrm{PSH}}(u, v)=t(x, y) \oplus I_{\mathrm{PSH}}(u, v) * I_{\mathrm{PSH}}(u, v)$. So, $I_{R}\left(x^{\prime}, y^{\prime}\right)=t(x, y) \oplus \Lambda(u, v)$ and $\Lambda$ is a delta-like function with a value of 1 at the origin and 0 elsewhere for an ideal case. However, previous studies have shown that the autocorrelation method dictates the sharpness of $\Lambda$ with sharpness increasing from the matched filter and phase-only filter toward a non-linear filter with minimum entropy [28-30]. Autocorrelation between positive functions results in substantial background noise. Therefore, in cases where the matched filter and phase-only filter are used, multiple camera shots are involved for generating complex (or at least real-bipolar) holograms $[17,18]$. The minimum value of the width of $\Lambda$ was obtained for the non-linear filter given by the diffraction limit $1.22 \lambda z_{\mathrm{s}} / D$.

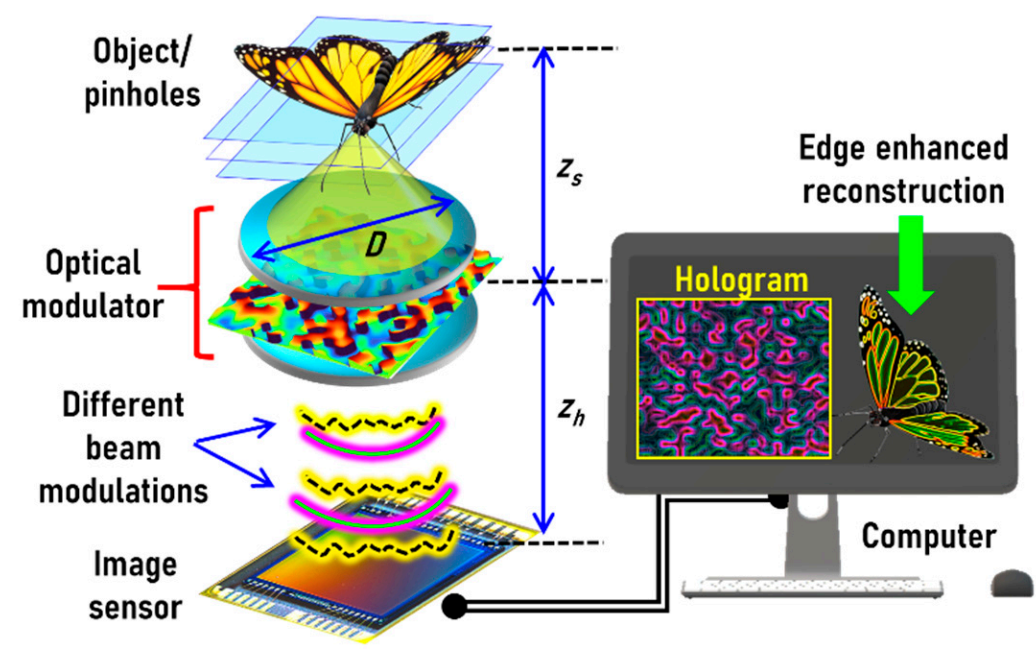

Figure 1. Optical configuration of spatially incoherent imager with coherent interference between two differently modulated object beams.

The aim of this study is to synthesize a special function-synthetic PSH $I_{S-P S H}$ from $I_{\mathrm{PSH}}$, which, when digitally cross-correlated with $I_{\mathrm{PSH}}$, generates a desired intensity distribution $I_{D}$. Since the main goal is to create an edge-enhanced image of the object, $I_{D}$ is chosen to have the donut-shaped spot [14]. Therefore, in the first step, the desired intensity distribution is simulated using the scalar diffraction formula with Fresnel or Fraunhofer approximation. More details of the diffraction functions and the intensity distributions with MATLAB code are given in [31]. The phase image of the spiral phase element with topological charge $L=1$ and the far-field diffraction patterns are shown in Figure 2. In the previous studies [16], higher topological charges are considered, but in this study, only $L=1$ is considered. 

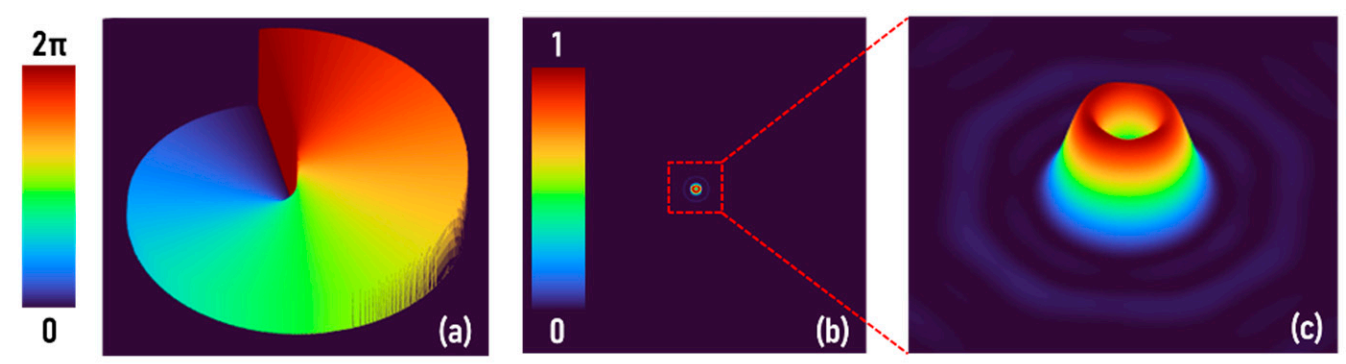

Figure 2. (a) Phase profile of a spiral phase plate with topological charge $L=1$. (b) Far-field diffraction pattern and (c) its magnified profile.

The iterative Fourier transform algorithm for synthesizing $I_{\mathrm{S}-\mathrm{PSH}}$ is shown in Figure 3. It is assumed that the Fourier transform of the synthetic PSH is a phase-only function $\exp (j \theta)$, and it is determined iteratively from the known functions: $I_{\mathrm{PSH}}$ and $I_{\mathrm{D}}$. The initial guess $\exp (j \theta)$ is a random phase function that is multiplied with the conjugated Fourier transform of the recorded $I_{\mathrm{PSH}}$ given as $\widetilde{I}_{\mathrm{PSH}}$, and the result is Fourier transformed. The resulting magnitude of the complex function is replaced by the desired magnitude distribution $I_{\mathrm{D}}$ a-priory calculated while the phase is retained, and the result is inversely Fourier transformed. The resulting complex function is multiplied by the inverse of $\widetilde{I}_{\mathrm{PSH}}{ }^{*}$, where a small value $\delta$ is added to guarantee that only non-zero values exist at the denominator. The resulting complex function is converted by $P_{2}$ to a pure phase function by eliminating its magnitude. The process is repeated until the intensity distribution in the image plane is close enough to $I_{\mathrm{D}}$. When the synthetic $\mathrm{PSH}$ is cross-correlated with the object hologram, an edge-enhanced image of the object is apparently obtained.

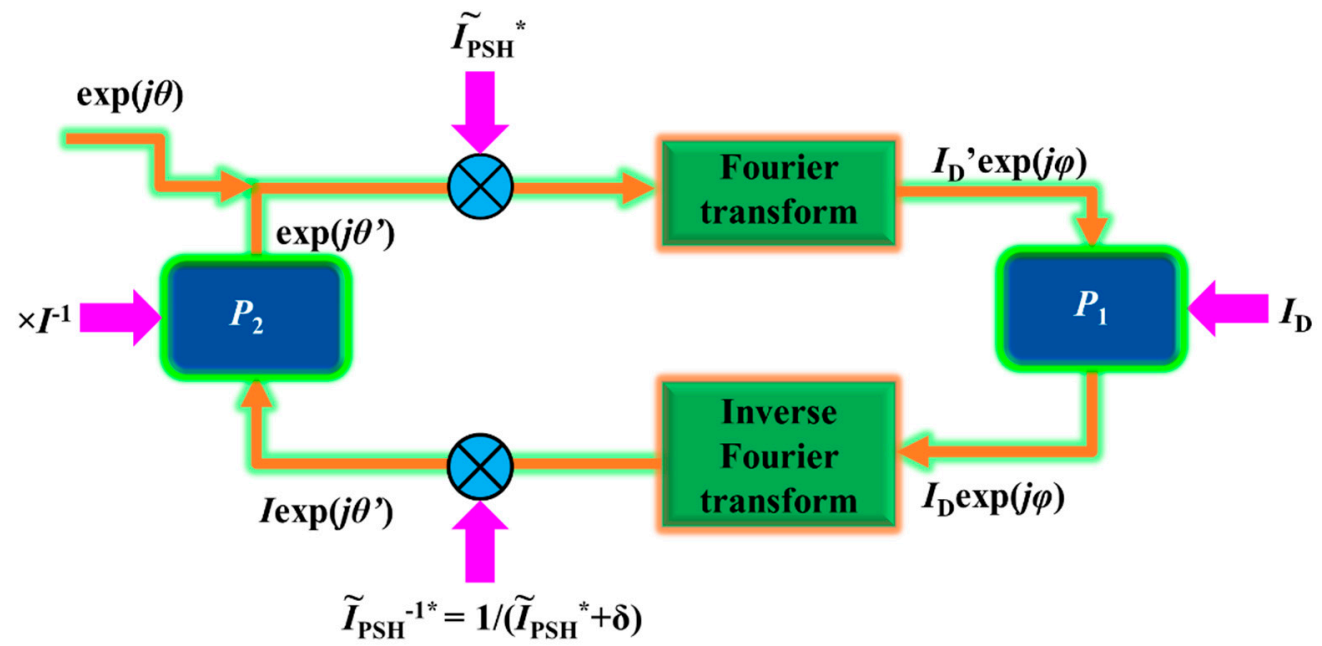

Figure 3. Schematic of the iterative algorithm for the generation of synthetic PSH. The symbol above $I_{\mathrm{PSH}}$ represents a Fourier transform operation, $\otimes$ represents a multiplication operation, and * represents complex conjugate.

A simulation was carried out using the word "Photonics" in Times New Roman fonts, as shown in Figure $4 \mathrm{a}$. A speckle pattern was generated and considered as $I_{\mathrm{PSH}}$, as shown in Figure $4 \mathrm{~b}$. The desired intensity response for a point source is given by Figure $2 \mathrm{~b}$. The object hologram corresponding to the "Photonics" object is shown in Figure 4c. The reconstructed image, obtained via cross-correlation with a phase-only filter, is shown in Figure $4 \mathrm{~d}$. The phase of the Fourier transform of the synthetic PSH is shown in Figure 4e. The edge-enhanced image obtained by cross-correlating the synthetic PSH with the object hologram is shown in Figure $4 \mathrm{f}$. The result of subtraction of Figure $4 \mathrm{f}$ from Figure $4 \mathrm{~d}$ $\left(I_{\text {Final }}=I_{R}-I_{R}{ }^{\prime}\right)$ after normalization generates an image with better contrast as shown in Figure $4 \mathrm{~g}$, where $I_{\text {Final }}$ is the contrast-enhanced image and $I_{R}$ and $I_{R}{ }^{\prime}$ are the reconstructions 
obtained with recorded PSH and synthetic PSH. Comparing the results of $I_{R}$ (Figure 4d) and $I_{\text {Final }}$ (Figure $4 \mathrm{~g}$ ), the improvement in contrast and sharpness is evident. Earlier studies on subtracting the edge-enhanced images from original images required experimental recording of the edge-enhanced images [32,33]. In the present study, the edge-enhanced images are obtained digitally from the same experimental data of the original image reconstruction.

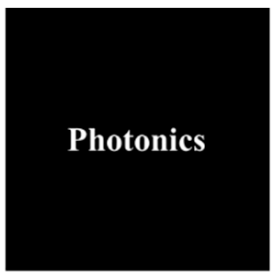

(a)

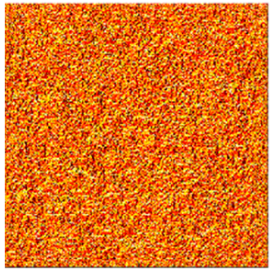

(e)

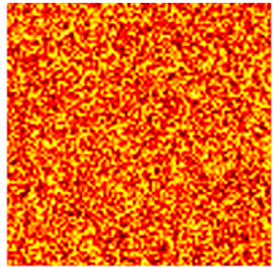

(b)

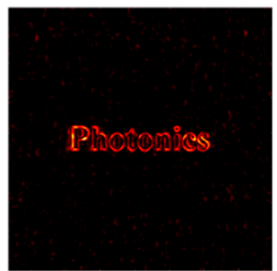

(f)

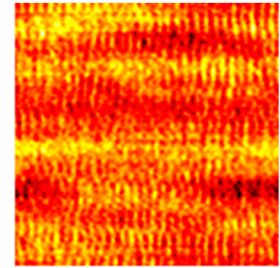

(c)

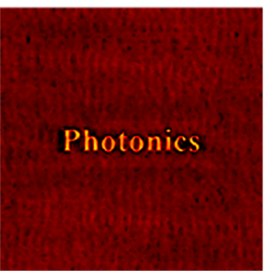

(g)

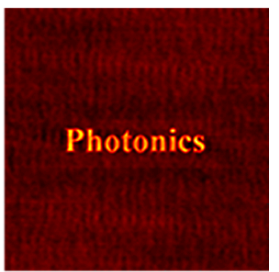

(d)
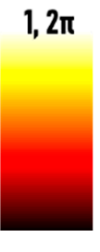

0

Figure 4. (a) Test object. (b) $I_{\mathrm{PSH}}$. (c) Object hologram. (d) Reconstructed image. (e) Phase of the synthetic PSH. (f) Edge-enhanced reconstruction and (g) contrast and resolution enhanced image.

\section{Results}

The edge and contrast enhancement studies were verified by implementing them in two types of imaging techniques, namely the FINCH and interferenceless CAI methods.

\subsection{FINCH}

FINCH was designed in a compact optical configuration with only one bifocal diffractive optical element (DOE) between the object and the image sensor, as shown in Figure 5a. The DOE was designed by randomly multiplexing two quadratic phase functions with focal distances of $2.5 \mathrm{~cm}$ and $5 \mathrm{~cm}$ and a diameter of $5 \mathrm{~mm}$. An electron beam resist PMMA $950 \mathrm{~K}$ was spin-coated on Indium Tin Oxide (ITO)-coated glass substrates to achieve the maximum possible efficiency $(\sim 40 \%)$ with a two-level structure [31]. A bifocal DOE with continuous phase variation can achieve a maximum efficiency of $100 \%$, theoretically. The elements were fabricated using electron beam lithography (RAITH $150^{2}$ ) and developed using Methyl Isobutyl Ketone (MIBK) and IsoPropyl Alcohol (IPA) in a 1:3 ratio. The optical microscope image of the fabricated DOE is shown in Figure 5b. A detailed discussion of the design and fabrication procedure is given in $[25,26]$.

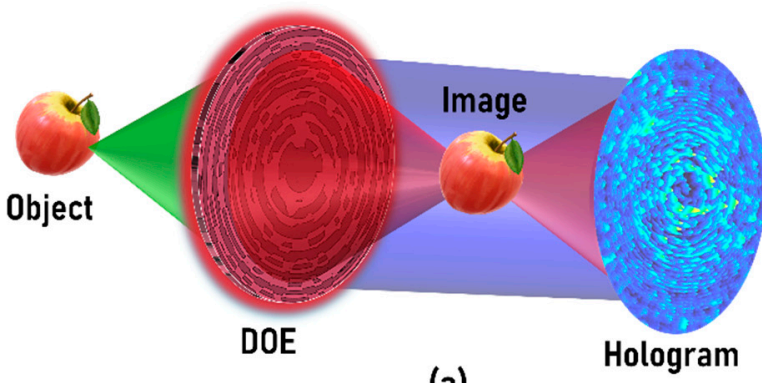

(a)

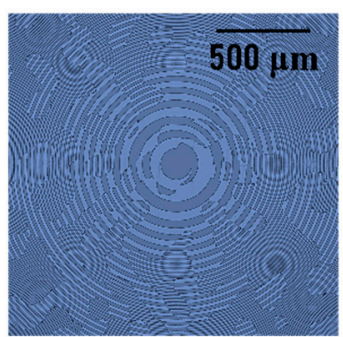

(b)

Figure 5. (a) Schematic of the compact optical configuration of FINCH and (b) optical microscope image of the bifocal DOE. 
The optical experiment was carried out using a high-power LED $\left(\mathrm{M} 617 \mathrm{~L} 3, \lambda_{\mathrm{c}}=617 \mathrm{~nm}\right.$, $\mathrm{FWHM}=18 \mathrm{~nm}$ ) and an image sensor (Thorlabs DCU223M, 1024 pixels $\times 768$ pixels, pixel size $=4.65 \mu \mathrm{m}$ ). In the first step, a pinhole with a diameter of $20 \mu \mathrm{m}$ was mounted at a distance of $5 \mathrm{~cm}$ from the DOE, and the sensor was located at $10 \mathrm{~cm}$ from the DOE. Therefore, part of the DOE $(f=5 \mathrm{~cm})$ collimated the incoming light while the other part $(f=2.5 \mathrm{~cm})$ experienced a $2 f$ configuration, resulting in a focusing of the light at $5 \mathrm{~cm}$ from the DOE, and it expanded from the focus to the sensor, satisfying the beam matching condition of FINCH [11]. The image of the self-interference PSH is shown in Figure 6a. A fungi sample on a glass plate used as the object was mounted in place of the pinhole, and the object hologram of Figure $6 \mathrm{~b}$ was recorded. The reconstructed image of the fungi using the non-linear filter is given as $I_{R}=\left|\mathcal{F}^{-1}\left\{\left|\widetilde{I}_{\mathrm{PSF}}\right|^{\alpha} \exp \left[i \arg \left(\widetilde{I}_{\mathrm{PSF}}\right)\right]\left|\widetilde{I}_{O}\right|^{\beta} \exp \left[-i \arg \left(\widetilde{I}_{o}\right)\right]\right\}\right|$, where the parameters $\alpha$ and $\beta$ are 0 and 0.7, respectively [34], as shown in Figure 6c. The direct image recorded at a distance of $5 \mathrm{~cm}$ from the DOE is shown in Figure $6 \mathrm{~d}$. Comparing Figure $6 \mathrm{c}, \mathrm{d}$, the higher resolution of FINCH is evident, indicating that the beam overlap condition has been met. Since the magnification is 1 for the direct imaging ( $2 f$ configuration), the original feature sizes can be obtained directly from the sensor pixel size. The synthetic PSH was generated using the iterative algorithm, while the phase of its Fourier transform is shown in Figure 6e. The edge and contrast-enhanced images are shown in Figure $6 \mathrm{f}$ (for $\alpha=1$ and $\beta=1$ ) and Figure 6g, respectively. Figure 6g appears not only sharper but also has a substantial reduction in background noise. Figure $6 \mathrm{~g}$ appears slightly smaller than Figure $6 c$, as the edges have been removed by the subtraction of Figure $6 f$ from Figure 6c. The plots of the line data extracted at different locations of the images are compared in Figure 6h. It is evident that the method enhances the reconstruction results of FINCH without additional experiments. Furthermore, the vortex beam's parameters can be adjusted directly during the digital synthesis, which is not possible in experiments without an active device (such as an SLM) or multiple optical elements.
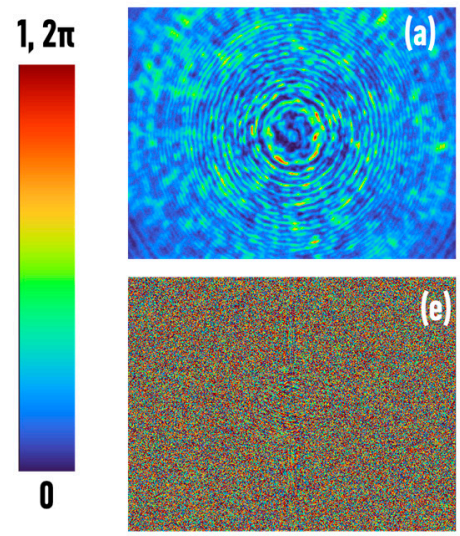
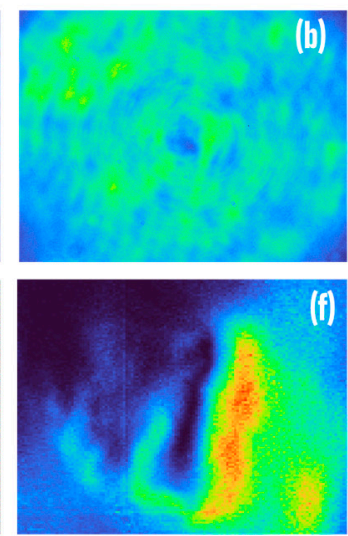
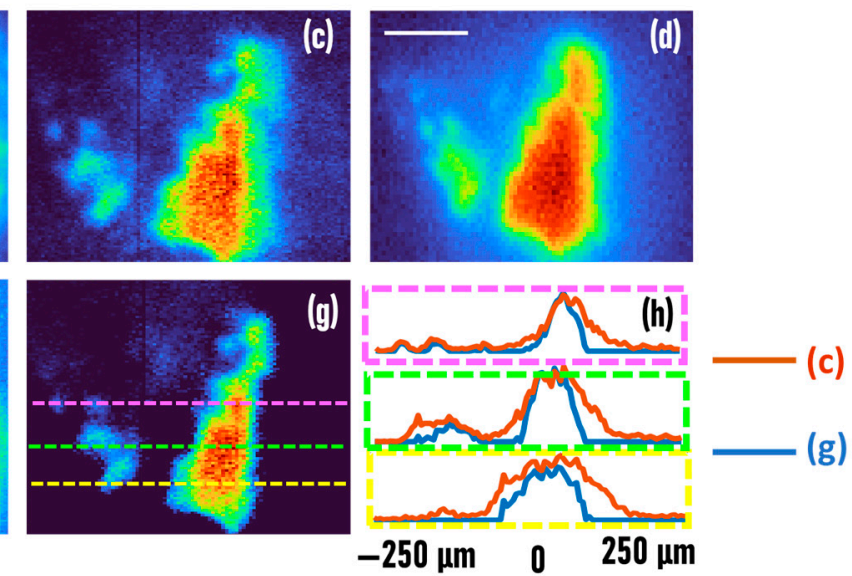

Figure 6. Experimentally recorded (a) PSH and (b) object hologram. (c) Reconstruction results by non-linear filter, (d) direct imaging. (e) Phase of the Fourier transform of synthetic PSH obtained from the iterative algorithm, (f) edge and (g) contrast-enhanced images. (h) Comparison between different line data of (c,g). The scale bar in (d) is $150 \mu \mathrm{m}$.

\subsection{CAI-Temporally Coherent}

The CAI was designed using a single diffractive element-a mask containing a quasirandom array of pinholes placed between the object and the sensor, as shown in Figure 7a. The pinhole array mask was designed with a total of 2000 pinholes with a diameter of $80 \mu \mathrm{m}$ randomly placed in an area of $8 \times 8 \mathrm{~mm}$ (Figure $7 \mathrm{~b}$ ). The location of the pinholes was adjusted using a search algorithm to minimize the background noise generated during autocorrelation [19]. The pinhole array mask was fabricated on chromium-coated glass 
plates using the Intelligent micropatterning SF100 XPRESS at the Melbourne Center for Nanofabrication.

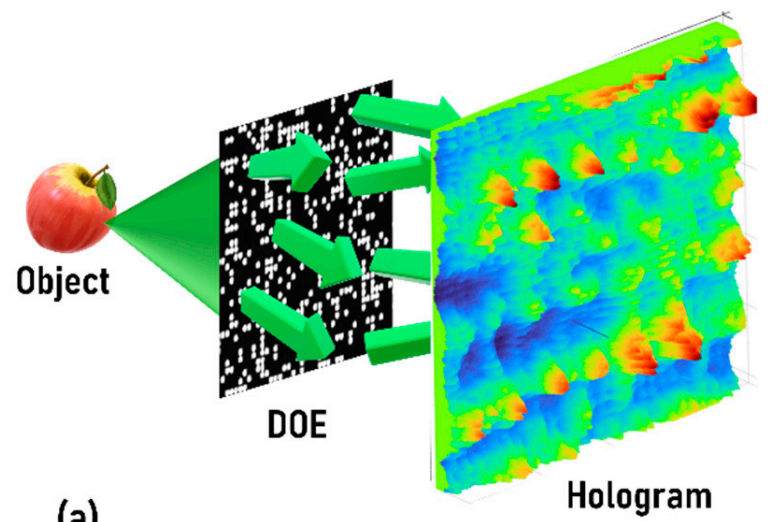

(a)

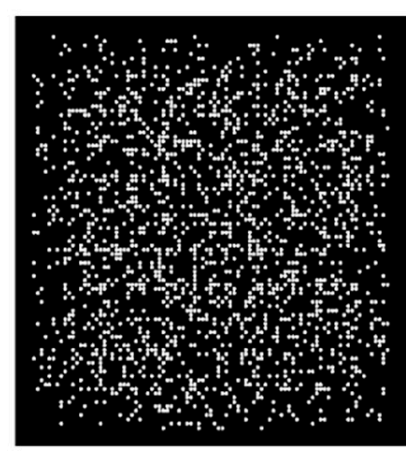

(b)

Figure 7. (a) Optical configuration of CAI with a pinhole array mask. (b) Image of the pinhole array mask.

The experiment was carried out by placing a pinhole with a diameter of $20 \mu \mathrm{m}$ at a distance of $10 \mathrm{~cm}$ from the pinhole array, and the sensor was placed at a distance of $10 \mathrm{~cm}$ from the pinhole array. The PSH was recorded for the high-power green $\operatorname{LED}(\lambda=532 \mathrm{~nm}$, $\Delta \lambda=1 \mathrm{~nm}$ ). The pinhole was replaced once by a USAF object (Group 2, elements 3 and 4) and later by the wing of an insect mounted to a slide glass, and two different object holograms were recorded. The iterative algorithm was used to calculate the synthetic PSH, and the object holograms were reconstructed using both the recorded PSH and the synthetic PSH. The reconstructed results were subtracted to obtain the contrast-enhanced images. The recorded PSH and object holograms, the results of reconstruction with a non-linear filter ( $\alpha=0$ and $\beta=0.8$ ), direct imaging results, the phase of the Fourier transform of the synthetic PSH, and the edge and contrast-enhanced images are shown in Figure 8 from left to right. It seems that the application of the algorithm improved the contrast notably. During the contrast enhancement procedure, the edges that control the sharpness were removed, resulting in an increase in the gap between different islands of object features.

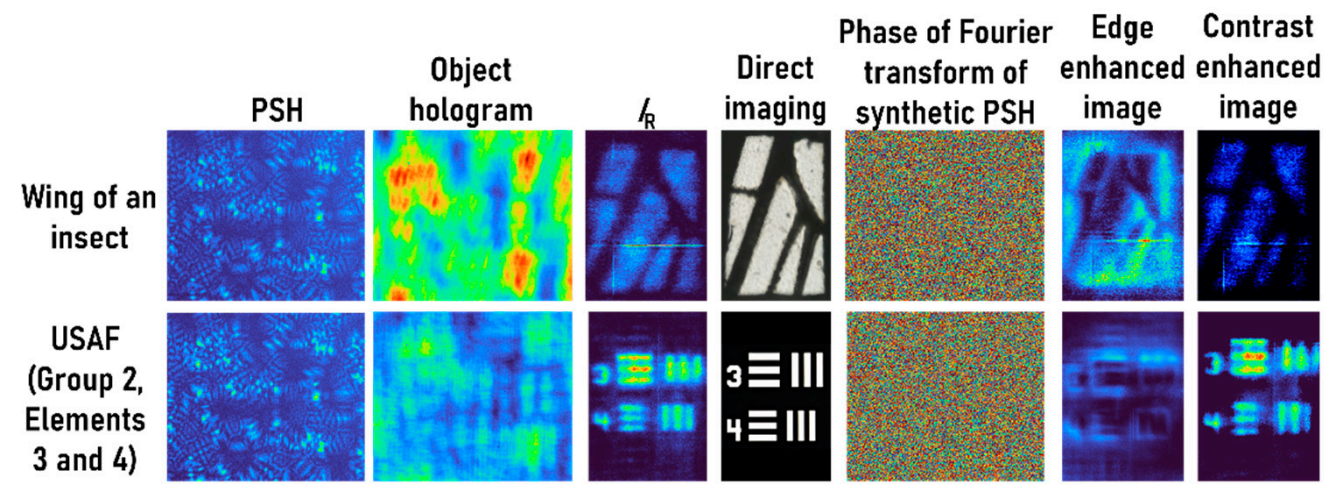

Figure 8. Images of the PSH, object hologram, reconstructed image $\left(I_{R}\right)$, direct imaging results, phase image of Fourier transform of synthetic PSH, edge and contrast-enhanced images for the wing of an insect and the USAF resolution target (Group 2, elements 3 and 4). 


\subsection{CAI-Temporally Low-Coherent}

The CAI imager was designed for high-speed imaging using an optical configuration shown in Figure 7a with a pinhole array similar to the one shown in Figure 7b. Instead of the regular camera, a high-speed camera (Phantom v2512, monochrome, $800 \times 1280$, $\Delta=28 \mu \mathrm{m}$ ) was used, and instead of static objects such as resolution targets, a high-speed dynamic event was imaged. In this case, a spark was generated by causing a rapid electrical discharge. More details of the spark event and experimental conditions can be found in [35]. The distance between the object and the random pinhole array mask was $5 \mathrm{~cm}$, and the distance between the random pinhole array mask and sensor was $15 \mathrm{~cm}$. The PSHs $(20 \mu \mathrm{m})$ were recorded using the red $(617 \mathrm{~nm}, \Delta \lambda=18 \mathrm{~nm})$ and green $(530 \mathrm{~nm}, \Delta \lambda=33 \mathrm{~nm})$ LEDs, respectively. The image of the spark recorded using a mobile camera is shown in Figure 9a. The images of the green PSH, object hologram, and the reconstructed image using a nonlinear filter $(\alpha=0$ and $\beta=0.85)$ are shown in Figure $9 \mathrm{~b}-\mathrm{d}$, respectively. The iterative algorithm was applied, and the phase of the Fourier transform of the synthetic PSH is shown in Figure 9e. The edge and contrast-enhanced images are shown in Figure 9f,g, respectively. Comparing Figure $9 d, g$, the contrast enhancement can be clearly seen.
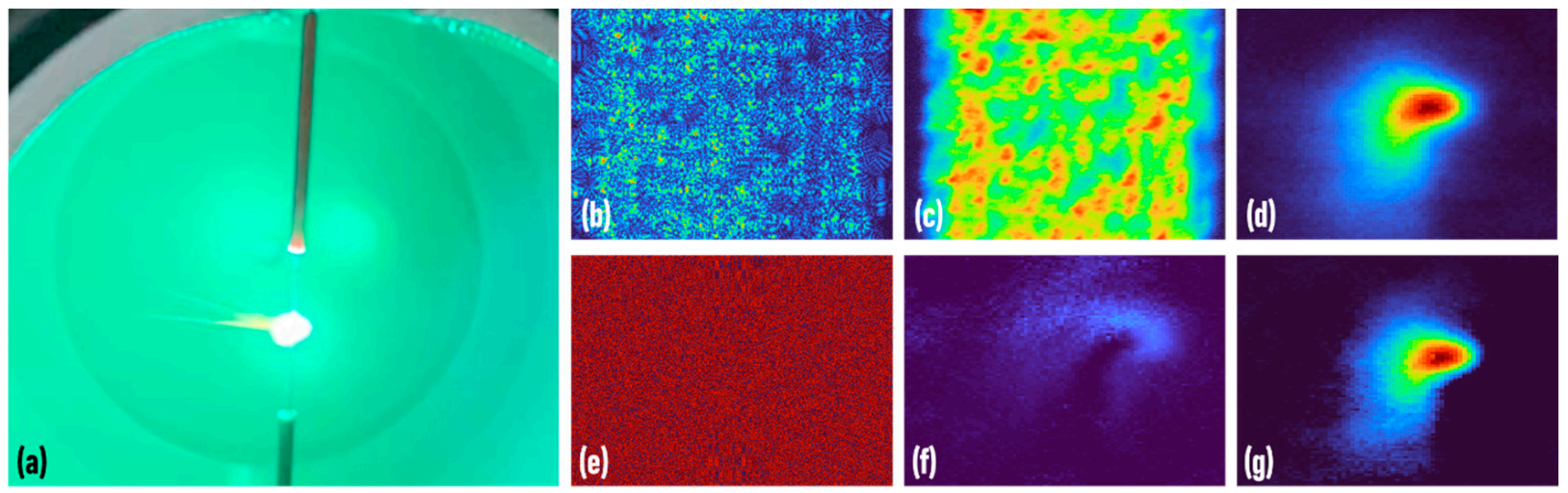

Figure 9. (a) Direct imaging of the spark. Images of the (b) PSH, (c) object hologram (d) reconstructed image using non-linear filter, (e) phase of the Fourier transform of the synthetic PSH, (f) edge, and (g) contrast-enhanced reconstructions.

\subsection{CAI-Temporally Incoherent}

The CAI was designed for white light with a quasi-random lens (QRL) between the object and the sensor [36]. The QRL was designed with a scattering ratio of 0.12 and object and image distances of $10 \mathrm{~cm}$ and fabricated using electron beam lithography (RAITH150 ${ }^{2}$ ) with a diameter of $5 \mathrm{~mm}$ (Figure 10a). Further details of the QRL fabrication can be found in [36]. A white light source (Fiber-Lite DC-950, Dolan-Jenner industries, full width at half maximum $\Delta \lambda \sim 270 \mathrm{~nm}$ ) was used for the experiment. A pinhole with a diameter of $100 \mu \mathrm{m}$ was used to record the PSHs. A USAF object, numeric digit 4, with a height of approximately $1 \mathrm{~mm}$, was used to record the object hologram. The images of the PSH, object hologram, and reconstructed image using a non-linear filter $(\alpha=0$ and $\beta=0.7)$ are shown in Figure $10 \mathrm{~b}-\mathrm{d}$, respectively. The iterative algorithm was implemented, and the phase of the Fourier transform of the synthetic PSH is shown in Figure 10e. The edge and contrast-enhanced images are shown in Figure 10f,g, respectively. The USAF object is shown in Figure $10 \mathrm{~h}$ for reference. Comparing Figure $10 \mathrm{~d}, \mathrm{~g}$, there is a significant reduction in background noise. 

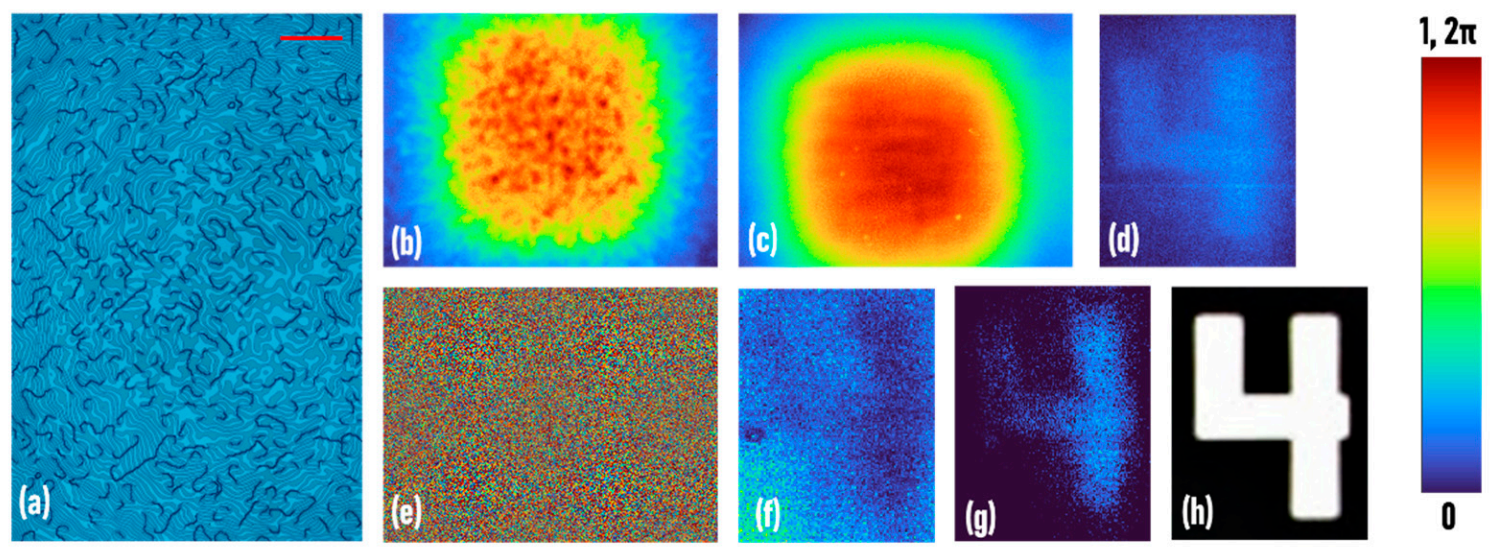

Figure 10. (a) Optical microscope image of the QRL. Images of the (b) PSH, (c) object hologram (d) reconstructed image using non-linear filter, (e) phase of the Fourier transform of the synthetic PSH, (f) edge, and (g) contrast-enhanced reconstructions. (h) Image of the object.

\section{Discussion}

A novel approach for edge and contrast enhancement has been carried out without the need for additional experiments with vortex filters, as it was done earlier. The approach has been demonstrated on different types of holographic systems (such as FINCH and $\mathrm{CAI}$ ) with different spectral configurations (such as temporally coherent, temporally lowcoherent, and temporally incoherent cases). For the above cases, depending upon the optical configuration and feature sizes of the object, the scaling factor of the vortex beam was altered to obtain the optimal performances in edge and contrast enhancement. It is necessary to run the iterative algorithm for different scaling factors of the vortex beam when edge and contrast enhancement is needed for a different object. It must be noted that such scaling adjustments are essential in the previous studies as well [13], which resulted in additional experiments. In the previous studies, in order to make the above-mentioned adjustment, it is necessary to experimentally change the focal distances. However, in the current approach, the adjustment can be digitally carried out on the computer.

Another significant aspect of this study is that the proposed approach is wavelengthindependent. With a method of [13], a perfect donut beam with a topological charge of 1 can be generated for only one wavelength, satisfying the complete phase profile $(0-2 \pi)$ for a particular wavelength and thus unable to match other wavelengths simultaneously. For other wavelengths, the intensity null at the center will not be obtained. Therefore, for low temporal coherence and temporal incoherence cases, the experimental approach using a vortex filter cannot be optimal. However, the proposed method generates a synthetic PSH for the recorded intensity distribution independently of the central wavelength and the spectral width. The number of iterations needed was about 100, and the total time needed for running the phase-retrieval algorithm in MATLAB software was around 13 seconds in a computer with i5-8250 with $1.6 \mathrm{GHz}$ and $1.8 \mathrm{GHz}$ processors and $8 \mathrm{~GB}$ RAM.

We reiterate that this method can be applied to all spatially incoherent, shift-invariant, indirect imaging systems in which the final image is reconstructed via cross-correlation with the recorded PSF. Even though the aim of the technique is to carry out edge and contrast enhancement, it also solved one main problem associated with indirect imagers that use scattering layers apertures [17-21] which is the background reconstruction noise. In the past, the background noise in the above indirect imagers was reduced by generating complex holograms and averaging over multiple intensity recordings [28]. In the present study, the reduced background reconstruction noise is obtained as a side effect of the contrast enhancement.

\section{Conclusions}

In this study, a novel approach involving an iterative algorithm was introduced to avoid additional optical experiments with vortex filters for edge and contrast enhancements 
in incoherent imaging systems. Different types of imagers, including FINCH and CAI with different temporal coherence conditions, were experimentally studied, and the feasibility of the approach was evaluated. The preliminary results are promising, with significant edge and contrast enhancements. The approach is expected to reduce the resources, cost, and time needed to carry out edge and contrast enhancement studies in holography and imaging systems.

Even though the method has been only demonstrated using spiral phase functions for the generation of vortex beams, the method can be extended to realize interesting optical fields such as Bessel beams, accelerating Airy beams, and other exotic beams, which are often difficult to synthesize experimentally. A similar algorithm can be used to introduce the beam characteristics of the above beams to the hologram without any optical experiments. We believe that this study has opened new possibilities to replace optical experiments for image enhancement if the point spread function of the system is experimentally recorded. Further investigation is needed to fully understand the capabilities and limitations of the new approach.

Author Contributions: Conceptualization, J.R., and V.A.; methodology, J.R.; Algorithm, J.R.; software, J.R. and V.A.; validation, V.A., S.H.N., T.K., D.P.L., and S.J.; resources, S.J.; DOE fabrication, V.A., T.K.; Bio samples preparation - D.P.L., and E.P.I.; writing—original draft preparation, V.A., J.R. and S.J.; writing-V.A, J.R and S.J.; visualization, J.R.; supervision, J.R., E.P.I., and S.J.; project administration, S.J.; funding acquisition, S.J. All authors have read and agreed to the published version of the manuscript.

Funding: NATO grant No. SPS-985048 and Australian Research Council (LP190100505). J.R. is supported by The Israel Science Foundation (ISF) (1669/16).

Data Availability Statement: All data generated or analyzed during this study are included in this published article.

Acknowledgments: The Nano Lab fabrication facility at Swinburne University of Technology is acknowledged.

Conflicts of Interest: The authors declare no conflict of interest.

\section{References}

1. Monnin, P.; Bulling, S.; Hoszowska, J.; Valley, J.F.; Meuli, R.; Verdun, F.R. Quantitative characterization of edge enhancement in phase contrast x-ray imaging. Med. Phys. 2004, 31, 1372-1383. [CrossRef]

2. Yue, Y.; Croitoru, M.M.; Bidani, A.; Zwischenberger, J.B.; Clark, J.W. Nonlinear multiscale wavelet diffusion for speckle suppression and edge enhancement in ultrasound images. IEEE Trans. Med. Imaging 2006, 25, 297-311.

3. Alonso, M.T.; López-Martínez, C.; Mallorquí, J.J.; Salembier, P. Edge enhancement algorithm based on the wavelet transform for automatic edge detection in SAR images. IEEE Trans. Geosci. Remote. Sens. 2010, 49, 222-235. [CrossRef]

4. Goodman, J.W. Introduction to Fourier Optics; Roberts and Company Publishers: Greenwood Village, CO, USA, 2005.

5. Saleh, B.E.A.; Teich, M.C. Fundamentals of Photonics; John Wiley \& Sons, Inc.: Hoboken, NJ, USA, 2019.

6. Sharma, M.K.; Joseph, J.; Senthilkumaran, P. Selective edge enhancement using anisotropic vortex filter. Appl. Opt. 2011, 50, 5279-5286. [CrossRef] [PubMed]

7. Khonina, S.N.; Kotlyar, V.V.; Shinkaryev, M.V.; Soifer, V.A.; Uspleniev, G.V. The phase rotor filter. J. Mod. Opt. 1992, 39, 1147-1154. [CrossRef]

8. Wang, J.; Zhang, W.; Qi, Q.; Zheng, S.; Chen, L. Gradual edge enhancement in spiral phase contrast imaging with fractional vortex filters. Sci. Rep. 2015, 5, 1-6. [CrossRef] [PubMed]

9. Rosen, J.; Brooker, G. Digital spatially incoherent Fresnel holography. Opt. Lett. 2007, 32, 912-914. [CrossRef]

10. Rosen, J.; Brooker, G. Non-Scanning Motionless Fluorescence Three-Dimensional Holographic Microscopy. Nat. Photonics 2008, 2, 190-195. [CrossRef]

11. Rosen, J.; Siegel, N.; Brooker, G. Theoretical and experimental demonstration of resolution beyond the Rayleigh limit by FINCH fluorescence microscopic imaging. Opt. Express 2011, 19, 26249-26268. [CrossRef] [PubMed]

12. Rosen, J.; Brooker, G. Fresnel incoherent correlation holography (FINCH)—A review of research. Adv. Opt. Technol. 2012, 1, 151-169. [CrossRef]

13. Bouchal, P.; Bouchal, Z. Selective edge enhancement in three-dimensional vortex imaging with incoherent light. Opt. Lett. 2012, 37, 2949-2951. [CrossRef]

14. Harris, M.; Hill, C.A.; Vaughan, J.M. Optical helices and spiral interference fringes. Opt. Commun. 1994, 106, 161-166. [CrossRef] 
15. Xu, T.; He, J.; Ren, H.; Zhao, Z.; Ma, G.; Gong, Q.; Yang, S.; Dong, L.; Ma, F. Edge contrast enhancement of Fresnel incoherent correlation holography $(\mathrm{FINCH})$ microscopy by spatial light modulator aided spiral phase modulation. Opt. Express 2017, 25, 29207-29215. [CrossRef]

16. Bu, Y.; Wang, X.; Li, Y.; Du, Y.; Gong, Q.; Zheng, G.; Ma, F. Tunable edge enhancement by higher-order spiral Fresnel incoherent correlation holography system. J. Phys. D Appl. Phys. 2021, 54, 125103. [CrossRef]

17. Vijayakumar, A.; Rosen, J. Interferenceless coded aperture correlation holography-A new technique for recording incoherent digital holograms without two-wave interference. Opt. Express 2017, 25, 13883-13896. [CrossRef] [PubMed]

18. Kumar, M.; Vijayakumar, A.; Rosen, J. Incoherent digital holograms acquired by interferenceless coded aperture correlation holography system without refractive lenses. Sci. Rep. 2017, 7, 11555. [CrossRef] [PubMed]

19. Anand, V.; Ng, S.H.; Maksimovic, J.; Linklater, D.; Katkus, T.; Ivanova, E.P.; Juodkazis, S. Single shot multispectral multidimensional imaging using chaotic waves. Sci. Rep. 2020, 10, 1-13. [CrossRef]

20. Rai, M.R.; Rosen, J. Noise suppression by controlling the sparsity of the point spread function in interferenceless coded aperture correlation holography (I-COACH). Opt. Express 2019, 27, 24311-24323. [CrossRef]

21. Antipa, N.; Kuo, G.; Heckel, R.; Mildenhall, B.; Bostan, E.; Ng, R.; Waller, L. DiffuserCam: Lensless single-exposure 3D imaging. Optica 2018, 5, 1-9. [CrossRef]

22. Wu, Y.; Sharma, M.K.; Veeraraghavan, A. WISH: Wavefront imaging sensor with high resolution. Light Sci. Appl. 2019, 8, 1-10. [CrossRef]

23. Sekko, E.; Thomas, G.; Boukrouche, A. A deconvolution technique using optimal Wiener filtering and regularization. Signal Process. 1999, 72, 23-32. [CrossRef]

24. Hojjatoleslami, S.A.; Avanaki, M.R.N.; Podoleanu, A.G. Image quality improvement in optical coherence tomography using Lucy-Richardson deconvolution algorithm. Appl. Opt. 2013, 52, 5663-5670. [CrossRef] [PubMed]

25. Anand, V.; Katkus, T.; Juodkazis, S. Randomly Multiplexed Diffractive Lens and Axicon for Spatial and Spectral Imaging. Micromachines 2020, 11, 437. [CrossRef] [PubMed]

26. Anand, V.; Katkus, T.; Lundgaard, S.; Linklater, D.P.; Ivanova, E.P.; Ng, S.H.; Juodkazis, S. Fresnel incoherent correlation holography with single camera shot. Opto-Electron. Adv. 2020, 3, 200004.

27. Anand, V.; Katkus, T.; Ng, S.H.; Juodkazis, S. Review of Fresnel incoherent correlation holography with linear and non-linear correlations. Chin. Opt. Lett. 2021, 19, 020501. [CrossRef]

28. Vijayakumar, A.; Kashter, Y.; Kelner, R.; Rosen, J. Coded aperture correlation holography system with improved performance. Appl. Opt. 2017, 56, F67-F77. [CrossRef]

29. Rai, M.R.; Vijayakumar, A.; Rosen, J. Non-linear adaptive three-dimensional imaging with interferenceless coded aperture correlation holography (I-COACH). Opt. Express 2018, 26, 18143-18154. [CrossRef]

30. Horner, J.L.; Gianino, P.D. Phase-only matched filtering. Appl. Opt. 1984, 23, 812-816. [CrossRef]

31. Vijayakumar, A.; Bhattacharya, S. Design and Fabrication of Diffractive Optical Elements with MATLAB; SPIE Press Book: Bellingham, WA, USA, 2017.

32. Tian, N.; Fu, L.; Gu, M. Resolution and contrast enhancement of subtractive second harmonic generation microscopy with a circularly polarized vortex beam. Sci. Rep. 2015, 5, 13580. [CrossRef]

33. Korobchevskaya, K.; Peres, C.; Li, Z.; Antipov, A.; Sheppard, C.J.; Diaspro, A.; Bianchini, P. Intensity weighted subtraction microscopy approach for image contrast and resolution enhancement. Sci. Rep. 2016, 6, 25816. [CrossRef]

34. Rai, M.R.; Vijayakumar, A.; Ogura, Y.; Rosen, J. Resolution enhancement in nonlinear interferenceless $\mathrm{COACH}$ with point response of subdiffraction limit patterns. Opt. Express 2019, 27, 391-403. [CrossRef] [PubMed]

35. Anand, V.; Ng, S.H.; Katkus, T.; Juodkazis, S. Spatio-spectral-temporal imaging of fast transient phenomena using a random array of pinholes. Adv. Photonics Res. 2021, 2, 2000032. [CrossRef]

36. Anand, V.; Ng, S.H.; Katkus, T.; Juodkazis, S. White light three-dimensional imaging using a quasi-random lens. Opt. Express 2021, 29, 15551-15563. [CrossRef] [PubMed] 